\title{
Sleeping position and electrocortical activity in low birthweight infants
}

\author{
R Sahni, K F Schulze, S Kashyap, K Ohira-Kist, W P Fifer, M M Myers

See end of article for authors' affiliations

\section{Correspondence to:} Dr Sahni, Department of Pediatrics, College of Physicians and Surgeons, Columbia University, 630 W 168th Street, New York, NY 10032, USA; rs62@columbia.edu

Accepted 5 February 2005 Published Online First 27 April 2005

\begin{abstract}
Objective: To evaluate the effects of prone and supine sleeping positions on electrocortical activity during active (AS) and quiet (QS) sleep in low birthweight infants.

Design: Randomised/crossover study.

Setting: Infant Physiology Laboratory at Children's Hospital of New York.

Patients: Sixty three healthy, growing, low birthweight (birth weight 795-1600 g) infants, 26-37 weeks gestational age.

Interventions: Six hour continuous two channel electrocortical recordings, together with minute by minute behavioural state assignment, were performed. The infants were randomly assigned to prone or supine position during the first three hours, and positions were reversed during the second three hours.

Outcome measures and results: Fast Fourier transforms of electroencephalograms (EEGs) were performed each minute and the total EEG power (TP), spectral edge frequency (SEF), absolute (AP) and relative (RP) powers in five frequency bands $(0.01-1.0 \mathrm{~Hz}, 1-4 \mathrm{~Hz}, 4-8 \mathrm{~Hz}, 8-12 \mathrm{~Hz}, 12-24 \mathrm{~Hz}$ ) were computed. Mean values for TP, SEF, $A P$, and RP in the five frequency bands in the prone and supine positions during $A S$ and $Q S$ were then compared. In the prone sleeping position, during AS, infants showed significantly lower TP, decreased AP in frequency bands $0.01-1.0 \mathrm{~Hz}, 4-8 \mathrm{~Hz}, 8-12 \mathrm{~Hz}, 12-24 \mathrm{~Hz}$, increased RP in 1-4 Hz, and a decrease in SEF. Similar trends were observed during QS, although they did not reach statistical significance. Conclusions: The prone sleeping position promotes a shift in EEG activity towards slower frequencies. These changes in electrocortical activity may be related to mechanisms associated with decreased arousal in the prone position and, in turn, increased risk of sudden infant death syndrome.
\end{abstract}

$\mathrm{D}$ espite the dramatic decline in the incidence of sudden infant death syndrome (SIDS) following worldwide education programmes, SIDS remains the major cause of death in infants 1-12 months of age. ${ }^{1}$ Accurate, prospective identification of individual infants likely to succumb is not yet possible, but several risk factors associated with this syndrome have been identified. Epidemiological data strongly relate prone sleeping position to greater risk of SIDS..$^{2-4}$ In the last decade, decreased use of the prone position for sleeping infants has been associated with almost $50 \%$ reduction in the incidence of SIDS. ${ }^{5}$ The physiological mechanisms that underlie SIDS remain unknown, and prospects for further reduction may be tied to a better understanding of the mechanisms linking prone sleep to SIDS.

The idea that SIDS and sleep are closely linked has been the prevailing view for many years, and abnormal state regulation has long been suspected to be a possible risk factor. ${ }^{7}$ In previous studies of preterm, ${ }^{89}$ term, $^{10}{ }^{11}$ and older infants, ${ }^{12}$ it was observed that, in the prone position, infants spent more time in quiet sleep (QS) and less time awake, but it still remains unclear why sleeping position influences sleep architecture. Recently, the prone sleeping position has been reported to be associated with higher arousal thresholds during both quiet and active sleep (AS) in healthy term infants. ${ }^{13}$ These position related differences in state distribution and arousal threshold could result from altered brain stem or cortical activity. Physiological functions influenced by brain stem activity, such as heart rate, ${ }^{14-19}$ blood pressure, ${ }^{20}$ and ventilation ${ }^{16}{ }^{19}$ are altered with body positioning. Furthermore, a deficit in arousal, responsiveness, or gasping is postulated as a necessary prerequisite for SIDS to occur. ${ }^{21}$ Although there is some evidence of electroencephalographic (EEG) abnormalities in at risk infants, ${ }^{22}{ }^{23}$ effects of prone sleeping position on quantitative differences in cortical activity has not been studied.
Low birth weight (LBW) associated with premature delivery stands out as one of the most significant risk factors for SIDS. ${ }^{24}$ This suggests that systematic studies of effects of sleeping position and sleep states on electrocortical activity in this high risk group could provide new insights into the understanding of SIDS. The objective of this study was to determine the effects of prone and supine sleeping position on electrocortical activity during QS and AS in healthy, growing LBW infants. Our general hypothesis was that in the prone sleeping position, as compared with supine, LBW infants would exhibit patterns of electrocortical activity that would be characteristic of deeper sleep. More specifically we proposed that, in the prone position, low frequency $(1-4 \mathrm{~Hz})$ activity would be increased, high frequency $(12-24 \mathrm{~Hz}$ ) would be decreased, and that this shift in power would be associated with a lower spectral edge frequency (SEF; defined as the frequency below which $95 \%$ of the power in a spectrum is found).

\section{METHODS}

\section{Study population}

The study population consisted of 63 healthy, growing LBW infants (birth weight 795-1600 g; 26-37 weeks gestational age), all of whom were enrolled in a prospective, double blind, controlled study of effects of quality of dietary energy on rate and composition of weight gain. The studies were approved by the institutional review board, and written consent was obtained from parents of all infants. All infants were being maintained in room air, were free of apnoea of prematurity, and were receiving no cardiac or respiratory drugs. None had sonographic evidence of central nervous

Abbreviations: $A P$, absolute power; $A S$, active sleep; $E E G$, electroencephalograph; LBW, low birth weight; QS, quiet sleep; RP, relative power; SEF, spectral edge frequency; SIDS, sudden infant death syndrome; TP, total power 
Table 1 Characteristics of study population $(n=63)$

\begin{tabular}{ll}
\hline Gestational age (weeks) & $30.5(2.5)(26-37)$ \\
Study age (weeks) & $34.5(1.5)(31-38)$ \\
Birth weight $(g)$ & $1264(232)(795-1600)$ \\
Study weight (g) & $1852(156)(1349-2235)$ \\
\hline \multicolumn{2}{l}{ Values are mean (SD) (range). }
\end{tabular}

system pathology at the time of the studies. Table 1 shows the characteristics of the infants.

\section{Experimental design}

Infants were studied in the Infant Physiology Laboratory at Children's Hospital of New York. Biweekly studies of about six hours duration were performed starting when the infant reached full enteral intake of $180 \mathrm{ml} / \mathrm{kg} /$ day. Each study comprised two sequential three hour periods of continuous recording of two-channel electrocortical activity. Simultaneously, behavioural assignment of sleep state was made for each minute of the study. Infants were randomly assigned to the supine or prone position for the first three hour epoch; the position was then reversed in the second three hour epoch. They remained in their assigned positions throughout the interfeeding period, and no further manipulations were performed.

\section{Experimental protocol}

Infants were brought to the laboratory at about 0730 at which time electrodes for recording electrocortical activity and vital signs were attached. They were then placed in a radiantly warmed, clear plastic incubator and maintained under thermoneutral conditions. No physical constraints such as swaddling were used. Studies began after the 0800 feed and continued until the 1400 feed. The studies were interrupted for the 1100 feed, after which sleeping position was changed. The volume and composition of the two feeds were identical.

\section{Measurement of electrocortical activity}

Six silver/silver chloride EEG electrodes were placed in a subset of the International 10-20 System montage. After the impedance of each electrode had been tested, the head was wrapped with a gauze headband to secure the electrodes. Two channels $\left(\mathrm{T}_{3}-\mathrm{O}_{1}\right.$ and $\left.\mathrm{T}_{4}-\mathrm{O}_{2}\right)$ of electrical potential were amplified using the Oxford Medilog 9000-II recording system. A low frequency filter of $0.5 \mathrm{~Hz}$ was used to minimise movement artefact, and a high frequency filter of $30 \mathrm{~Hz}$ was used to ensure that aliasing did not occur when the signal was digitised at $100 \mathrm{~Hz}$. The analogue outputs from both channels were digitised at $100 \mathrm{~Hz}$ with a Labmaster 12-bit analogue to digital converter and stored on a microcomputer.

\section{Assessment of sleep state}

Behaviour codes were assigned each minute using a scoring system developed and validated in our laboratory. ${ }^{25}$ Briefly, AS was coded if one or more rapid eye movements were observed during the minute. In addition to small body

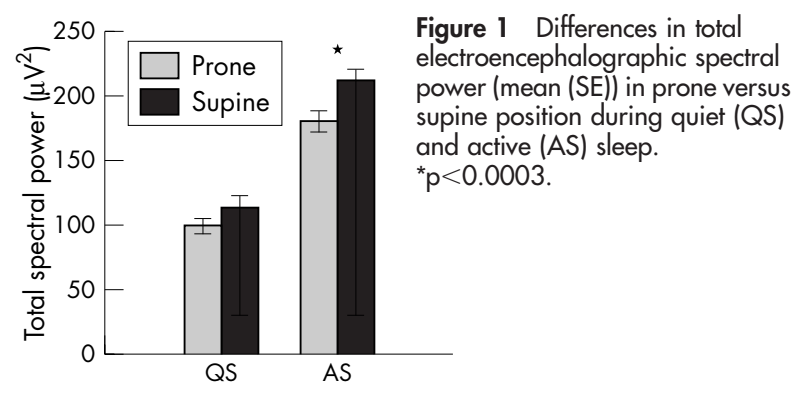

movements typical of AS, movements of whole extremities and the torso were seen in this state. QS was designated when the infant was asleep without rapid eye movements and appeared "rag-doll" floppy and relaxed; movements were limited to startles and non-nutritive sucking or jaw jerks. Indeterminate state was coded when small body movements were observed, without rapid eye movements. Codes were also assigned for awake, crying, and feeding periods.

\section{Data analysis}

The EEG was subjected to spectral analysis. Fast Fourier transforms were performed on the first 40.96 seconds $\left(2^{12}\right.$ data points) of each minute of EEG data. Total EEG power (TP) was partitioned into five frequency bands $(0.01-1.0 \mathrm{~Hz}$, 1-4 Hz, 4-8 Hz, 8-12 Hz, and 12-24 Hz). SEF and absolute power (AP) in each of the bands was computed. Relative power (RP) for each frequency band was computed as a percentage of AP in each frequency band over the TP. TP, AP, and RP for each frequency band, and SEF were averaged across the two channels. Data from each study were then segregated for different sleep states in prone and supine positions. The results from the two studies performed for each infant were then averaged, yielding a single mean value. The infant means for $\mathrm{TP}, \mathrm{AP}$, and RP in the five frequency bands, and SEF in the prone versus supine positions during QS, AS, and awake state were compared using paired $t$ tests. Further, the effect of development (age and weight) on the size of these postural differences was evaluated using correlation analysis.

\section{RESULTS}

As shown in fig 1, in the prone sleeping position, there was a significant decrease in TP when compared with the supine position during AS (mean (SD) 180.70 (61.51) v 211.68 $\left.(80.50) \mu V^{2}, p<0.0003\right)$. A similar direction of effect was seen for QS but the difference was not significant (100.52 (39.38) $\left.\nu 114.44(66.51) \mu \mathrm{V}^{2}, \mathrm{p}=0.24\right)$. Significantly lower AP was observed in the prone sleeping position during AS for all but the $1-4 \mathrm{~Hz}$ band. Effects during QS were in a similar direction but were not significant (table 2).

As shown in table 3, the prone sleeping position was also associated with significantly higher RP in the $1-4 \mathrm{~Hz}$ frequency band during AS. Again, prone versus supine differences in RP during QS were in a similar direction but were not significant.

Consistent with the increase in RP in the $1-4 \mathrm{~Hz}$ band in the prone position, prone sleeping led to a shift in SEF to lower frequencies in AS (mean (SD) 4.69 (1.06) $v 5.17$ (1.17) $\mathrm{Hz}, \mathrm{p}<0.000 \mathrm{l}$; fig 2). The change in SEF in QS was in the same direction $(4.24(0.87) v 4.44(1.01) \mathrm{Hz}, \mathrm{p}=0.06)$. There were no significant positional differences in SEF, TP, AP, and RP during the awake state.

With increasing postconceptional age, significant amplification in prone-supine differences were observed for AP in frequency ranges $1-4,4-8,8-12$, and $12-24 \mathrm{~Hz}$ during AS $(r>0.28, \mathrm{p}<0.03)$. Prone-supine differences in SEF, TP, and RP did not vary with age. Weight showed no significant relation to any of the postural differences in EEG spectral variables during QS or AS.

\section{DISCUSSION}

The hypothesis guiding our study was that, in the prone position, EEG activity would be shifted in a direction that would be indicative of deeper sleep-that is, $1-4 \mathrm{~Hz}$ activity would be higher and 12-24 Hz activity would be lower in the prone versus the supine position. When infants were judged to be in QS, position had no significant effect on either AP or RP in any frequency band. In AS this hypothesis was supported.

However, this interpretation requires examination of both $\mathrm{AP}$ and RP and consideration of the possible impact of 
Table 2 Absolute electroencephalographic spectral power $\left(\mu \mathrm{V}^{2}\right)$ in five frequency bands in prone and supine positions during quiet and active sleep

\begin{tabular}{|c|c|c|c|c|c|c|}
\hline \multirow{2}{*}{$\begin{array}{l}\text { Frequency } \\
\text { band (Hz) }\end{array}$} & \multicolumn{3}{|l|}{ Quiet sleep } & \multicolumn{3}{|l|}{ Active sleep } \\
\hline & Prone & Supine & p Value & Prone & Supine & p Value \\
\hline $0.01-1$ & $82.27(36.37)$ & $95.19(59.77)$ & 0.26 & $158.24(58.04)$ & $187.63(75.94)$ & $<0.0003$ \\
\hline $1-4$ & $14.30(5.14)$ & $14.99(6.85)$ & 0.28 & $17.72(5.90)$ & $18.30(6.37)$ & 0.08 \\
\hline $4-8$ & $1.67(0.92)$ & $1.85(1.45)$ & 0.34 & $1.68(0.64)$ & $1.90(0.89)$ & $<0.0005$ \\
\hline $8-12$ & $0.73(0.38)$ & $0.80(0.51)$ & 0.29 & $0.69(0.28)$ & $0.81(0.43)$ & $<0.005$ \\
\hline $12-24$ & $0.74(0.37)$ & $0.83(0.54)$ & 0.23 & $1.09(0.44)$ & $1.29(0.67)$ & $<0.001$ \\
\hline
\end{tabular}

movement artefact. As hypothesised, in AS, AP in the 12$24 \mathrm{~Hz}$ band was lower in the prone position. However, the expected increase in absolute $\mathrm{l}-4 \mathrm{~Hz}$ activity was not found. We propose that this increase is due to interference from low frequency artefact associated with movements. This, in concert with reduced movement in the prone position, obscured position related differences in $1-4 \mathrm{~Hz}$ activity. The AP in 0.01-1 Hz nearly doubled in AS as compared with QS. This increase probably results from contributions of movement artefact to activity in low frequency bands. Consistent with this interpretation is the finding that absolute $1-4 \mathrm{~Hz}$ activity was not higher in QS as would be expected. However, when state differences in the effect of movement artefact were reduced by expressing power relative to TP, higher levels of $1-4 \mathrm{~Hz}$ activity in QS were apparent.

AP in the $0.01-1 \mathrm{~Hz}$ band was about $19 \%$ greater in the supine versus the prone position. Although we did not quantify body or eye movements during the study, it is our impression that infants move less in the prone position. Thus, as with state, effects of body position on absolute levels of low frequency activity may also be masked by movement artefact. However, dividing by TP (which is primarily due to low frequencies) resolved the expected position effect on 1$4 \mathrm{~Hz}$ activity. In contrast, as there was little or no movement artefact at higher frequencies, dividing 12-24 Hz activity by the movement-contaminated TP eliminated the effects of position that were apparent in AP.

Despite successful campaigns throughout the world promoting the sleeping of infants in the supine or side position rather than in the prone position and reduction in incidence of SIDS, the mechanisms by which the prone sleeping position increases the risk of SIDS remains elusive. A number of reasons have been suggested as to why the prone position may present an increased risk of SIDS. Sleeping in the prone position has been shown to increase the amount of time spent sleeping, ${ }^{1526}$ particularly time spent in $\mathrm{QS}^{8-12}$ and is associated with a reduced responsiveness to a variety of arousal stimuli, both external and internal. ${ }^{12}{ }^{12}$ This decreased propensity to arouse from sleep has been shown in both QS $^{14}$ and AS. ${ }^{13} 141928$ Prone sleeping is also associated with many changes in control of autonomic cardiac function, as shown by elevated heart rate in QS and AS, ${ }^{14-20}$ decreased heart rate variability, ${ }^{16} 18$ 28-30 increased orthosympathetic tone, ${ }^{13} 1820$ decreased parasympathetic tone, ${ }^{29}$ and lower blood pressure. $^{20}$ Thus the combination of impaired arousal and altered autonomic nervous system function are probably intertwined in such a way as to increase risk of death in this sleeping position.

Whether sleeping position related changes in arousal are associated with changes in cortical function as reflected in EEG characteristics is not known. There is some evidence of accelerated appearance of several developmental characteristics of the sleep EEG, such as increase in spectral power in the 12-15 Hz band during QS at 4-8 weeks postnatal age in siblings of SIDS victims as compared with 8-12 weeks in control infants. ${ }^{22}$ Schectmann and colleagues have shown increased integrated delta amplitude in the early morning hours in SIDS siblings relative to control infants at 34 months postnatal age, suggesting increased arousal thresholds in the morning in SIDS siblings. ${ }^{23}$ These abnormalities of sleep EEG patterns are of special interest, as most SIDS deaths occur during sleep periods, ${ }^{31}$ and a failure to arouse from a life threatening challenge encountered during sleep may play a role. ${ }^{32}{ }^{33}$ Of particular interest is slow wave deltathat is, $1-4 \mathrm{~Hz}$-EEG activity which is not only a prominent characteristic of QS but is present as early as 32 week gestational age $\mathrm{e}^{34}$ and increases in amount and amplitude during QS throughout later gestation and early postnatal life. ${ }^{35}{ }^{36}$ The presence of slow wave activity during sleep is indicative of deeper sleep (higher arousal thresholds) in adult humans and animals. ${ }^{37}{ }^{38}$ It is likely that infants are also more resistant to arousal when their sleep is characterised by slow wave activity. Decreases in TP, decreases in AP in higher frequencies, increases in RP in the low frequency band (1-4 Hz), and a shift in the SEF to lower frequencies are major distinguishing features of QS versus AS. Prone sleeping position led to significant changes in all four of these variables in the direction of QS, suggesting that the prone position promotes a deeper AS and may be related to a higher arousal threshold. These findings are especially important, as LBW infants, of 30-39 weeks postconceptional age, spend $74 \%$ of their time in AS. $^{39}$

There is limited information on spectral analysis of EEG activity in preterm infants. The frequency distribution of the

Table 3 Relative electroencephalographic spectral power (\%) in five frequency bands in prone and supine positions during quiet and active sleep

\begin{tabular}{|c|c|c|c|c|c|c|}
\hline \multirow{2}{*}{$\begin{array}{l}\text { Frequency } \\
\text { band }(\mathrm{Hz})\end{array}$} & \multicolumn{3}{|l|}{ Quiet sleep } & \multicolumn{3}{|l|}{ Active sleep } \\
\hline & Prone & Supine & p Value & Prone & Supine & p Value \\
\hline $0.01-1$ & $80.62(6.08)$ & $81.37(6.10)$ & 0.59 & $87.00(3.56)$ & $87.96(3.55)$ & $<0.003$ \\
\hline $1-4$ & $15.32(5.00)$ & $14.68(5.16)$ & 0.58 & $10.27(3.03)$ & $9.24(2.90)$ & $<0.0003$ \\
\hline $4-8$ & $1.76(0.73)$ & $1.71(0.71)$ & 0.80 & $0.97(0.32)$ & $0.93(0.31)$ & 0.12 \\
\hline $8-12$ & $0.79(0.35)$ & $0.77(0.32)$ & 0.80 & $0.40(0.14)$ & $0.40(0.16)$ & 0.60 \\
\hline $12-24$ & $0.80(0.33)$ & $0.79(0.32)$ & 0.08 & $0.64(0.23)$ & $0.65(0.28)$ & 0.70 \\
\hline
\end{tabular}

Values are mean (SD). 


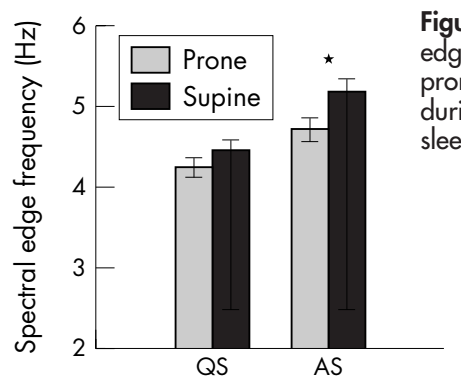

EEG activity and the SEF in our study infants was very similar to previously published observations of LBW infants. ${ }^{4041}$ Schramm and colleagues ${ }^{42}$ reported a reduction in the AP in various frequency ranges $(0.4-19.5 \mathrm{~Hz})$ during periods of central apnoeas in preterm and term infants. This reduction from baseline was present in the time periods before and after manifestation of an apnoea. The authors suggest that these lower voltages of electrical activity represent parallel deficits in arousal reaction. Similar to their findings, we found lower spectral powers in the prone sleeping position during AS. These sleep related changes in power during prone positioning may result from vestibular mediated influences through the cerebellum to thalamic synchronising means..$^{43}$ This process, in turn, may inhibit incoming messages and deprive the cerebral cortex of signals from the outside world and account for impaired arousal response in the prone position. Any impairment in arousability or associated shift in the electrocortical activity towards that characteristic of QS when infants are in the prone position could contribute to the final pathway to SIDS. However, most would agree that an arousal deficit failure alone would not result in SIDS unless it was paired with a lethal challenge.

Many questions concerning the importance of arousal and SIDS remain unanswered. During arousal from sleep, heart rate, blood pressure, and ventilation are increased, and, importantly, a behavioural response is evoked, allowing movement away from a life threatening stimulus. Our data lend support to a "failure to arouse" hypothesis for SIDS in that LBW infants, sleeping in the prone position exhibit a shift in the electrocortical activity towards the slower frequencies. Further advances in this area could potentially contribute to further reduction in SIDS incidence and earlier detection of infants at highest risk.

\section{ACKNOWLEDGEMENTS}

This work was supported by United States Public Health Service Grants HD 13063, HD 27564, HD 32774, and a clinical research center grant RR00645.

\section{Authors' affiliations}

R Sahni, K F Schulze, S Kashyap, K Ohira-Kist, Department of Pediatrics, College of Physicians and Surgeons, Columbia University, New York, NY 10032, USA

W P Fifer, M M Myers, Department of Psychiatry, College of Physicians and Surgeons

This study was performed in the Infant Physiology Laboratory at Children's Hospital of New York, Columbia University, New York, NY.

Competing interests: none declared

\section{REFERENCES}

1 MacDorman MF, Minino AM, Strobino DM, et al. Annual summary of vital statistics: 2001. Pediatrics 2002;110:1037-52.

2 Consensus: a scientific review of the association between prone sleeping position and sudden infant syndrome. J Paediatr Child Health $1991 ; 27: 323-4$.

3 Dwyer T, Posonby AL, Newman NM, et al. Prospective cohort study of prone position and sudden infant syndrome. Lancet 1991;337:1244-7.
4 Mitchell EA, Tuohy PG, Brunt JM, et al. Risk factors of sudden infant death syndrome following the prevention campaign in New Zealand: a prospective study. Pediatrics 1997; 100:835-40

5 Adams EJ, Chavez GF, Steen D, et al. Changes in the epidemiologic profile of sudden infant death syndrome as rates decline among California infants: 1990-1995. Pediatrics 1998;102:1445-51.

6 Willinger M, Ko CW, Hoffman HJ, et al. Factors associated with caregivers' choice of infant sleep position, 1994-1998: the National Infant Sleep Position Study. JAMA 2000;283:2135-42.

7 Harper RM, Kinney HC, Fleming PJ, et al. Seep influences on homeostatic functions: implications for sudden infant death syndrome. Respir Physiol 2000; 1 19:123-32.

8 Myers MM, Fifer WP, Schaeffer L, et al. Effects of sleeping position and time after feeding on the organization of sleep/wake states in prematurely born infants. Sleep 1998;21:343-9.

9 Sahni R, Saluja D, Schulze KF, et al. Quality of diet, body position, and time after feeding influence behavioral states in low birth weight infants. Pediatr Res 2002;52:399-404

10 Brackbill Y, Douthitt TC, West H. Psychophysiologic effects in the neonate of prone versus supine placement. J Pediatr 1973;82:82-4.

11 Amemiya F, Vos JE, Prechtl HF. Effects of prone and supine position on heart rate, respiratory rate and motor activity in full term infants. Brain Dev 1991;3:148-54.

12 Kahn A, Rebuffat E, Sottiaux $M$, et al. Arousal induced by proximal esophageal reflux in infants. Sleep 1991;14:39-42.

13 Franco P, Pardou A, Hassid S, et al. Auditory arousal thresholds are higher when infants sleep in the prone position. J Pediatr 1998;132:240-3.

14 Horne RS, Ferens D, Watts AM, et al. The prone sleeping position impairs arousability in term infants. J Pediatr 2001;138:811-16.

15 Kahn A, Groswasser J, Sottiaux M, et al. Prone and supine body position and sleep characteristics in infants. Pediatrics 1993;91:1112-15.

16 Sahni R, Schulze KF, Kashyap S, et al. Body position, sleep states, and cardiorespiratory activity in developing low birth weight infants. Early Hum Dev 1999;54:197-206

17 Goto K, Mirmiran M, Adams MM, et al. More awakenings and heart rate variability during supine sleep in preterm infants. Pediatrics 1999;103:603-9.

18 Sahni R, Schulze KF, Kashyap S, et al. Postural differences in cardiac dynamics during quiet and active sleep in low birth weight infants. Acta Paediatr 1999;88:1396-401.

19 Galland BC, Bolton DP, Taylor BJ, et al. Ventilatory sensitivity to mild asphyxia: prone versus supine sleep position. Arch Dis Child 2000;83:423-8.

20 Chong A, Murphy N, Matthews T. Effect of prone sleeping on circulatory control in infants. Arch Dis Child 2000;82:253-6.

21 Wulbrand H, McNamara F, Thach BT. Suppression of sigma spindle electroencephalographic activity as a measure of transient arousal after spontaneous and occlusion-evoked sighs and startles. Pediatr Res 1998;44:767-73.

22 Sterman MB, McGinty DJ, Harper RM, et al. Developmental comparison of sleep EEG power spectral patterns in infants at low and high risk for sudden death. Electroencephalogr Clin Neurophysiol 1982;53:166-81.

23 Schechtman VL, Harper RK, Harper RM. Aberrant temporal patterning of slow-wave sleep in siblings of SIDS victims. Electroencephalogr Clin Neurophysiol 1995;94:95-102.

24 Hoffman HJ, Damus K, Hillman L, et al. Risk factors for SIDS: Results of the National Institute of Child Health and Human Development SIDS cooperative epidemiological study. In: Schwartz PJ, Southall DP, Valdes Dapena M, eds. The sudden infant death syndrome: cardiac and respiratory mechanisms and interventions. 533rd ed. New York: Annals of the New York Academy of Science, 1988:13-30.

25 Stefanski M, Schulze KF, Bateman D, et al. A scoring system for states of sleep and wakefulness in term and preterm infants. Pediatr Res 1984;18:58-63.

26 Groswasser J, Simon T, Scaillet S, et al. Reduced arousals following obstructive apneas in infants sleeping prone. Pediatr Res 2001;49:402-6.

27 Newman NM, Trinder JA, Phillips KA, et al. Arousal deficit: mechanism of the sudden death syndrome? Aust Paediatr J 1989;25:196-201.

28 Galland BC, Reeves G, Taylor BJ, et al. Sleep position, autonomic function, and arousal. Arch Dis Child Fetal Neonatal Ed 1998;78:F189-94.

29 Franco P, Groswasser J, Sottiaux M, et al. Decreased cardiac responses to auditory stimulation during prone sleep. Pediatrics 1996;97:174-8.

30 Ariagno RL, Mirmiran M, Adams MM, et al. Effect of position on sleep, heart rate variability, and QT interval in preterm infants at 1 and 3 months' corrected age. Pediatrics 2003;111:622-5.

31 Peterson DR. Evolution of epidemiology of sudden infant death syndrome. Epidemiol Rev 1980;2:97-112.

32 Harper RM, Leake B, Hoffman H, et al. Periodicity of sleep states is altered in infants at risk for the sudden infant death syndrome. Science $1981 ; 213: 1030-2$

33 Hunt CE. Impaired arousal from sleep: relationship to sudden infant death syndrome. J Perinatol 1989:9:184-7.

34 Dreyfus-Brisac C. Ontogenesis of sleep in human prematures after 32 weeks of conceptional age. Dev Psychobiol 1970;3:91-121.

35 Watanabe K, Iwase K, Hara K. Development of slow-wave sleep in low-birth weight infants. Dev Med Child Neurol 1974;16:23-31.

36 Sterman MB, Harper RM, Havens B, et al. Quantitative analysis of infant EEG development during quiet sleep. Electroencephalogr Clin Neurophysiol 1977;43:371-85

37 Rechtschaffen A, Hauri P, Zeitlin M. Auditory awakening thresholds in REM and NREM sleep stages. Percept Mot Skills 1966;22:927-42.

38 Neckelmann D, Ursin R. Sleep stages and EEG power spectrum in relation to acoustical stimulus arousal threshold in the rat. Sleep 1993; 16:467-77.

39 Sahni R, Schulze KF, Stefanski M, et al. Methodological issues in coding sleep states in immature infants. Dev Psychobiol 1995;28:85-101. 
40 Bell AH, McClure BG, McCullagh PJ, et al. Spectral edge frequency of the EEG in healthy neonates and variation with behavioural state. Biol Neonate 1991;60:69-74.

41 Scher MS, Sun M, Steppe DA et al. Comparisons of EEG sleep state-specific spectral values between healthy full-term and preterm infants at comparable postconceptional ages. Sleep 1994;17:47-51.
42 Schramm D, Scheidt B, Hubler A, et al. Spectral analysis of electroencephalogram during sleep-related apneas in pre-term and term born infants in the first weeks of life. Clin Neurophysiol 2000;111:1788-91.

43 Steriade $M$. The corticothalamic system in sleep. Front Biosci 2003;8:d878-99.

\section{IMAGES IN NEONATAL MEDICINE}

\section{Prenatal three dimensional ultrasound detection of linear nevus sebaceous syndrome}

doi: $10.1136 /$ adc.2003.038984

A ull term, male neonate was born to a 38 year old mother by uncomplicated vaginal delivery. Amniocentesis had been performed, and a chromosome study revealed a normal male karyotype at 11 weeks gestation. A prenatal three dimensional sonogram showed two skin taglike lesions over the left orbital region at 28 weeks gestation (fig 1).

Physical examination of the neonate found linear, well demarcated, yellowish, hairless plaques located on the forehead, scalp, and facial region. Two nodules over the lateral canthus and upper eyelid of the left eye resulted in ectropion (fig 2) There were no other cutaneous lesions. Magnetic resonance imaging of the brain showed hemimegalencephaly. The baby suffered a seizure attack about two weeks after birth.

Linear nevus sebaceous syndrome is a rare sporadic oculoneurocutaneous disorder, consisting of a spectrum of abnormalities involving the skin, central nervous system, eyes, and other systems. The major clinical manifestations include linear nevus sebaceous, seizure, and mental retardation. The aetiology of this disorder is still not identified. ${ }^{1}$ The major finding on brain image is hemimegalencephaly, which is characterised by congenital overgrowth of one cerebral hemisphere ipsilateral to the skin lesions. The affected brain has essentially no function, and is often associated with hemiparesis, early onset seizures, mental retardation, hemimacrocephaly, and severe encephalopathy clinically. ${ }^{1-3}$ Prenatal diagnosis is difficult but a fetal sonogram, especially a three dimensional image, may be useful for early detection of linear nevus sebaceous syndrome. ${ }^{45}$

S-H Lien, M-L Hsu, Y-S Yuh, C-M Lee Department of Pediatrics, Tri-Service General Hospital, Taipei, Taiwan, ROC

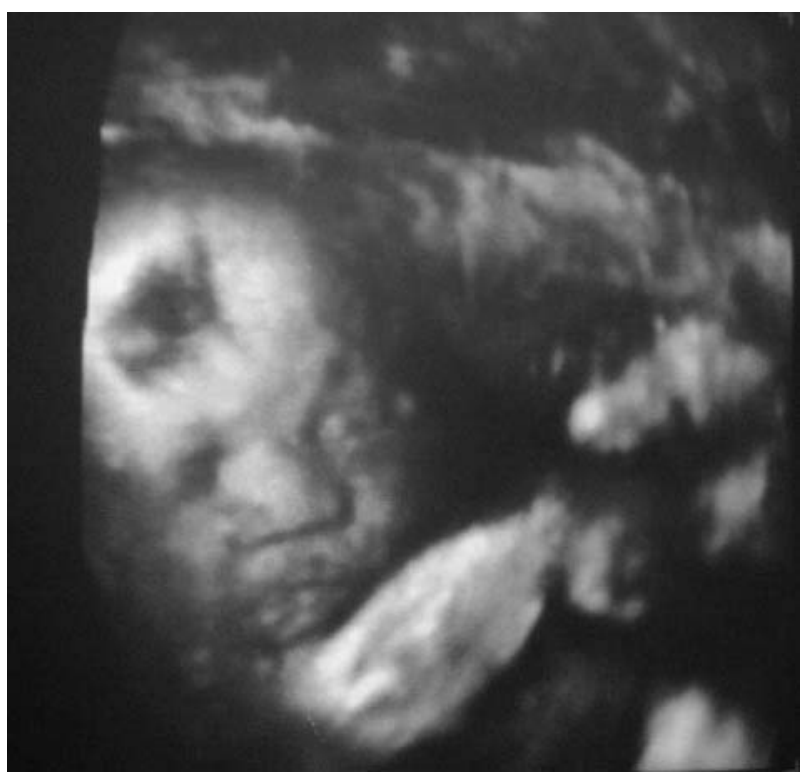

Figure 1 Skin tag-like lesions over left orbital region seen on three dimensional image at 28 weeks gestation.
C-C Chen, P-Y Chang

Department of Pediatrics, Armed Forces Sung Shan Hospital, Taipei, Taiwan,

ROC

C-Y Chou

Department of Obstetrics \& Gynecology, Armed Forces Sung Shan Hospital

Correspondence to: Dr Lee, Department of Pediatrics, Tri-Service General Hospital, No 325, Cheng-Kung Road, Sec 2, Neihu 114, Taipei, Taiwan,

ROC; lcmpin@yahoo.com.tw

Parental consent was obtained for publication of figure 2

Competing interests: none declared

\section{REFERENCES}

1 Alfonso I, Howard C, Lopez PF, et al. Linear nevus sebaceous syndrome. A review. J Clin Neuro-ophthalmol 1987;7:170-7.

2 Herman TE, Siegel MJ. Hemimegalencephaly and linear nevus sebaceous syndrome. J Perinatol $2001 ; 21: 336-8$.

3 Brodsky MC, Kincannon JM, Nelson-Adesokan P, et al. Oculocerebral dysgenesis in the linear nevus sebaceous syndrome. Ophthalmology 1997; 104:497-503.

4 Sweeney WJ, Kuller JA, Chescheir NC, et al. Prenatal ultrasound findings of linear nevus sebaceous and its association with cystic adenomatoid malformation of the lung. Obstet Gynecol 1994;83:860-2.

5 Nowaczyk MJ, Mernagh JR, Bourgeois JM, et al. Antenatal and postnatal findings in encephalocraniocutaneous lipomatosis. Am J Med Genet 2000;91:261-6.

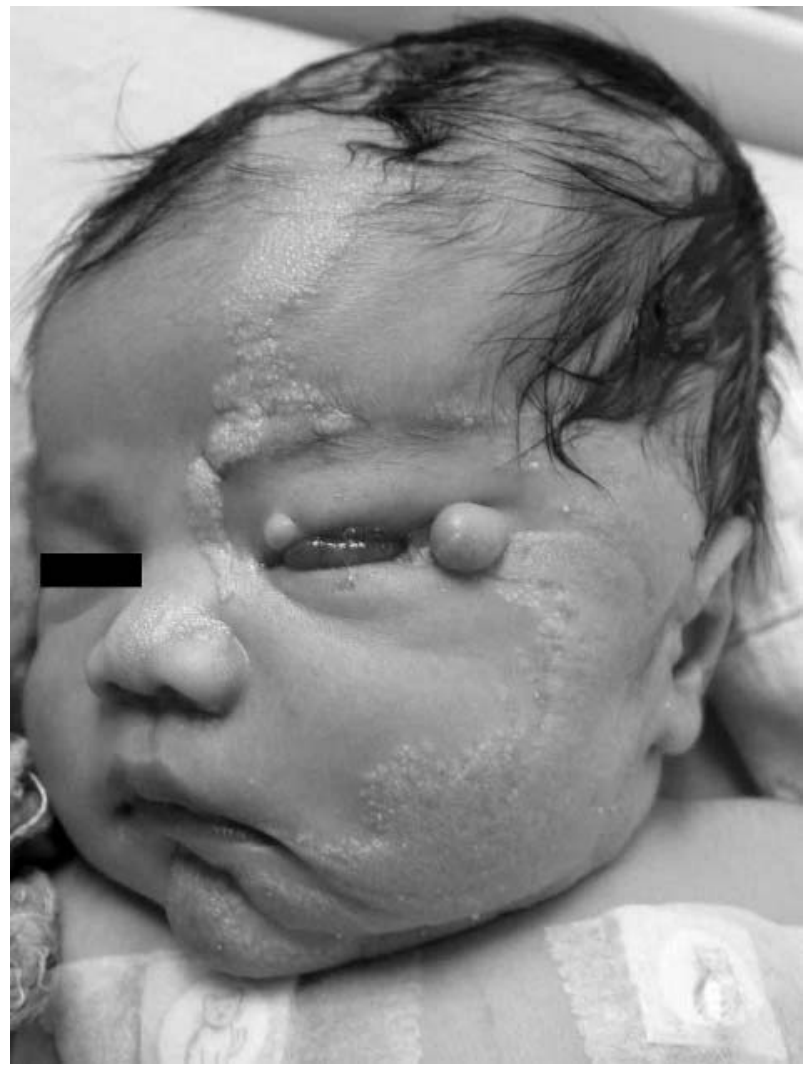

Figure 2 Linear, well demarcated, slightly raised, yellowish, hairless plaques located on the left side of the forehead, the parietal region of the scalp, face, and chin. Congenital ectropion caused by two nodules over the lateral canthus and upper eyelid can be seen. 